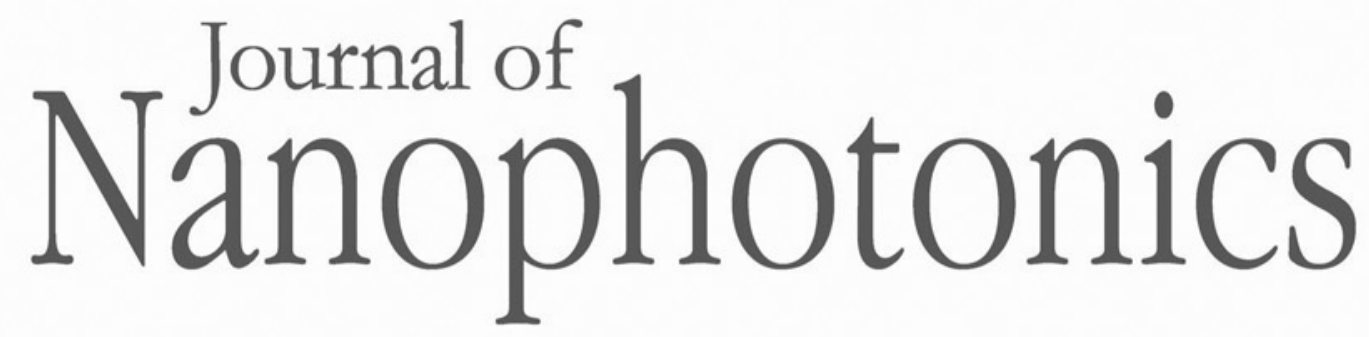

Nanophotonics.SPIEDigitalLibrary.org

\title{
Kinetic magnetoplasmons in graphene and their excitation by laser
}

Chuan Sheng Liu

Vipin K. Tripathi 


\title{
Kinetic magnetoplasmons in graphene and their excitation by laser
}

\author{
Chuan Sheng Liu and Vipin K. Tripathi*, ${ }^{\dagger}$ \\ University of Macao, Chao Kuang Piu College, Institute for Applied Physics and \\ Material Engineering, Macao, China
}

\begin{abstract}
A transverse magnetic field in graphene, together with the high speed of Dirac electrons moving with Fermi velocity, gives rise to a set of collective modes, viz., kinetic magnetoplasmonic modes, two-dimensional equivalent of Bernstein modes, with frequencies in between the harmonics of electron cyclotron frequency. We develop a Vlasov theory of these modes in a moderate magnetic field, including finite gyroradius effects, and study their excitation by laser through linear mode conversion, facilitated by grating or periodic ribbons. At $k \rho \rightarrow 0$ (where $k$ is the wave number and $\rho$ is the gyroradius of electrons), the magnetoplasmonic modes have frequencies near the harmonics of electron cyclotron frequency. The frequencies rise with wave number, attain maxima in the vicinity of the next cyclotron harmonic, and then fall off. In highmobility graphene, with ribbons or grating of appropriate ripple wave number, a normally impinged laser coverts a significant fraction of its power into magnetoplasmons, reducing the laser transmissivity as observed in experiments. () The Authors. Published by SPIE under a Creative Commons Attribution 3.0 Unported License. Distribution or reproduction of this work in whole or in part requires full attribution of the original publication, including its DOI. [DOI: 10.1117/1.JNP.11.036015]
\end{abstract}

Keywords: laser; plasmonics; optical phenomena; terahertz; graphene.

Paper 17047 received Apr. 20, 2017; accepted for publication Jul. 10, 2017; published online Aug. 22, 2017.

\section{Introduction}

One important property of graphene $\mathrm{e}^{1-8}$ is that all its Dirac electrons, irrespective of their energy, have the same speed; $v_{F}=10^{8} \mathrm{~cm} / \mathrm{s}$ as energy versus momentum relation is linear. When a transverse magnetic field is applied, the electrons are localized in circular orbits and graphene supports a variety of manetoplasmonic modes. Roldan et al. ${ }^{9,10}$ have developed a formalism of these two-dimensional (2-D) Bernstein modes based on Dirac equation using random phase approximation at intense magnetic fields, where Landau levels have energies comparable to Fermi energy.

Basov et al. ${ }^{1}$ have discussed plasmons in graphene in the presence of a transverse magnetic field in the limit of small Larmor radius. Yan et al. ${ }^{11,12}$ have detected bulk like and edge like magnetoplasmon modes in graphene discs. Crassee et al. ${ }^{13}$ have observed terahertz magnetoplasmons in graphene, mounted on substrate terrace or wrinkles, through a sharp dip in the transmission coefficient of normally impinged radiation. It is believed that at the dip frequency, laser undergoes linear mode conversion into magnetoplasmons. They have also observed Faraday rotation (FR) of an optical wave passing through single layer graphene. Martinez and Jalil ${ }^{14}$ have developed quantum formalism of FR. In the limit of $\hbar \omega_{c} \ll \varepsilon_{F}$ (where $\omega_{c}$ is the electron cyclotron frequency, $\varepsilon_{F}$ is the Fermi energy and $\hbar$ is the reduced Planck's constant), the effect of Landau quantization is unimportant.

Theoretical studies on linear mode conversion of laser into surface plasmons are largely limited to unmagnetized case. Mikhailov ${ }^{15}$ has developed a formal analytical theory of laser mode coupling to a plasmonic mode in an unmagnetized 2-D structure. Lee and Degertekin ${ }^{16}$ have developed a coupled wave analysis of multilayered grating structures, but the results are obtained

\footnotetext{
*Address all correspondence to: Vipin K. Tripathi, E-mail: tripathivipin@yahoo.co.in

${ }^{\dagger}$ Permanent address: Indian Institute of Technology Delhi, Physics Department, New Delhi, India.
} 


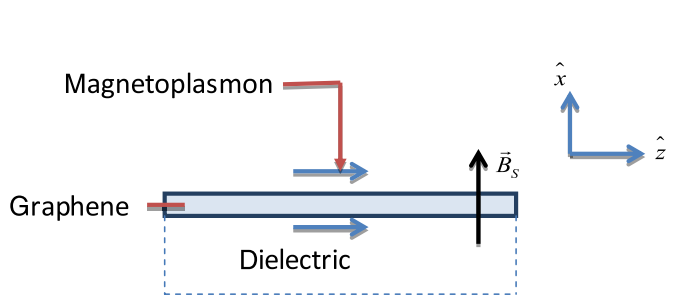

(a)

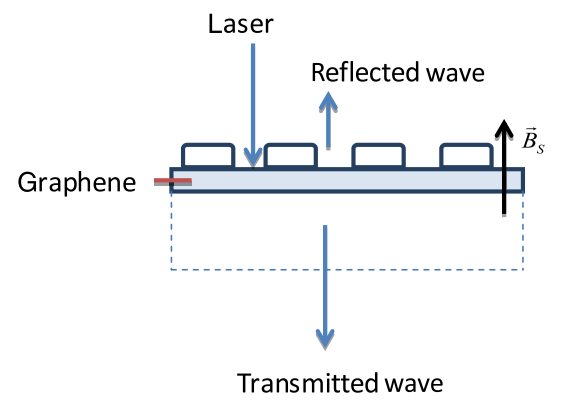

(b)

Fig. 1 (a) Schematic of graphene mounted on a dielectric slab. A transverse static magnetic field $\vec{B}_{S}$ is applied along $\hat{x}$. Magnetoplasmonic mode propagates along $\hat{z}$ while its amplitude falls off with $|x|$. (b) Graphene is embedded with periodic ribbons. A laser is normally impinged on graphene.

numerically for reflected diffraction efficiency. Gao et al. ${ }^{17}$ have carried numerical simulations of linear mode conversion.

In this paper, we develop Vlasov formalism of magnetoplasmons in graphene mounted on a dielectric placed in a transverse static magnetic field and study their excitation via linear mode conversion. Our treatment is restricted to moderate magnetic fields such that the Landau level energy separation is smaller than Fermi energy. We find that the finite gyroradius effects give rise to multiple magnetoplasmonic Bernstein modes, similar to the ones predicted by Roldan et al. at intense magnetic fields. A suitable grating built in the dielectric substrate or an areal density ripple, facilitates linear mode conversion. The grating creates a large wave number Fourier component of the laser field that creates an electron density perturbation, driving the plasmonic wave. Finer grating would generate Bernstein modes. One may mention that though the electron dynamics in graphene is strongly correlated and one normally uses Dirac theory to deduce optical conductivity, Boltzmann's equation reasonably describes transport properties. ${ }^{2,18}$ Furthermore, we may add that graphene plasmons can also be excited by electron beams. ${ }^{19,20}$ Batrakov and Maksimenkov ${ }^{20}$ have studied theoretically the excitation of terahertz surface wave over a system of unmagnetized graphene layers by a nonrelativistic electron beam. They obtain spatial growth rate of the order of $0.2 \mathrm{~cm}^{-1}$ at $30 \mathrm{THz}$ in eight layered graphene, using a $10-\mathrm{keV}$ electron beam. The frequency of the wave can be tuned by beam energy as well as by sheet separation and doping.

In Sec. 2, we study magnetoplasmonic modes of graphene in a transverse magnetic field including finite gyroradius effects. In Sec. 3, we study the linear mode conversion of radiation into magnetoplasmons in graphene with periodic ribbons. In Sec. 4, we study the mode conversion by a grating. In Sec. 5, we discuss the results.

\section{Magnetoplasmons}

Consider a graphene film mounted on a dielectric of relative permittivity $\varepsilon_{g}$ (cf., Fig. 1). There exists a transverse static magnetic field $B_{s} \hat{x}$. The graphene is characterized by free electrons of areal density $N_{0}^{0}$, energy-momentum relation $\varepsilon=v_{F} p$, velocity $\vec{\nu}=\partial \varepsilon / \partial \vec{p}=v_{F} \vec{p} / p$, and 2-D equilibrium distribution function

$$
f_{0}^{0}=\frac{1 /\left(2 \pi^{2} \hbar^{2}\right)}{e^{\left(\varepsilon-\varepsilon_{F}\right) / T}+1}
$$

where $\varepsilon_{F}$ is the Fermi energy, $\nu_{F}$ is the Fermi velocity $\left(\simeq 10^{8} \mathrm{~cm} / \mathrm{s}\right), T$ is the temperature in energy units, $\hbar$ is the reduced Planck's constant. At a low temperature to which we confine here, $\varepsilon_{F} / T \gg 1, N_{0}^{0}=2 \pi \int_{0}^{\infty} f_{0}^{0} p \mathrm{~d} p=\varepsilon_{F}^{2} / 2 \pi \hbar^{2} \nu_{F}^{2}$.

We perturb the equilibrium by a space charge mode of potential

$$
\phi=A e^{-k_{z} x} e^{-i\left(\omega t-k_{z} z\right)} \quad \text { for } \quad x>0, \quad \phi=A e^{k_{z} x} e^{-i\left(\omega t-k_{z} z\right)} \quad \text { for } \quad x<0,
$$


in compliance with the Poisson's equation $\nabla^{2} \phi=0$ (valid everywhere except in the graphene layer) and the continuity of $\phi$ at $x=0$. To incorporate the effect of graphene layer, we write the 2-D Vlasov equation for free electrons

$$
\frac{\partial f}{\partial t}+\vec{v} \cdot \nabla f-e(\vec{E}+\vec{v} \times \vec{B}) \cdot \frac{\partial f}{\partial \vec{p}}=0
$$

where $-e$ is the electron charge and $\vec{E}, \vec{B}$ are the electric and magnetic fields. In the presence of the space charge mode, we write $f=f_{0}^{0}+f_{\omega}^{L}$ and linearize the Vlasov equation

$$
\frac{\partial f_{\omega}^{L}}{\partial t}+\vec{v} \cdot \nabla f_{\omega}^{L}-e \vec{v} \times \vec{B}_{s} \cdot \frac{\partial f_{\omega}^{L}}{\partial \vec{p}} \equiv \frac{\mathrm{d} f_{\omega}^{L}}{\mathrm{~d} t}=-e \nabla \phi \cdot \frac{\partial f_{0}^{0}}{\partial \vec{p}},
$$

to obtain the linear perturbation $f_{\omega}^{L}$

$$
f_{\omega}^{L}=-i e A \frac{1}{p} \frac{\partial f_{0}^{0}}{\partial p} \int_{-\infty}^{t} \vec{k} \cdot \vec{p}^{\prime} e^{-i\left(\omega t^{\prime}-k_{z} z^{\prime}\right)} \mathrm{d} t^{\prime}
$$

where $z^{\prime}, \vec{p}^{\prime}$ refer to position and momentum of electron at time $t^{\prime}$ and the integration is to be carried over the unperturbed trajectory of electron in the static magnetic field, governed by the equation of motion

$$
\frac{\mathrm{d} \vec{p}^{\prime}}{\mathrm{d} t^{\prime}}=-e \vec{v}^{\prime} \times \vec{B}_{s}=-\frac{e v_{F}}{p} \vec{p}^{\prime} \times \vec{B}_{s}
$$

Under the conditions that at $t^{\prime}=t$, electron has position and momentum $y^{\prime}=y, z^{\prime}=z$, $p_{z}^{\prime}=p_{z}=p \cos \theta, p_{y}^{\prime}=p_{y}=-p \sin \theta$ (where $\theta$ is the gyrophase angle), Eq. (5) gives

$$
\begin{aligned}
p_{z}^{\prime} & =p \cos \left[\omega_{c}\left(t^{\prime}-t\right)+\theta\right], \quad p_{y}^{\prime}=-p \sin \left[\omega_{c}\left(t^{\prime}-t\right)+\theta\right], \\
z^{\prime} & =z+\frac{v_{F}}{\omega_{c}}\left\{\sin \left[\omega_{c}\left(t^{\prime}-t\right)+\theta\right]-\sin \theta\right\}, \\
y^{\prime} & =y+\frac{v_{F}}{\omega_{c}}\left\{\cos \left[\omega_{c}\left(t^{\prime}-t\right)+\theta\right]-\cos \theta\right\},
\end{aligned}
$$

where $\omega_{c}=e B_{s} v_{F} / p$. Using Eq. (6) and employing the Bessel function identity, $e^{i \alpha \sin \theta}=\sum_{l} J_{l}(\alpha) e^{i l \theta}$, we obtain from Eq. (4)

$$
f_{\omega}^{L}=\frac{e \phi}{v_{F}} \frac{\partial f_{0}^{0}}{\partial p} \sum_{l} \sum_{l^{\prime}} \frac{l \omega_{c}}{\omega-l \omega_{c}} J_{l}\left(\frac{k_{z} v_{F}}{\omega_{c}}\right) J_{l^{\prime}}\left(\frac{k_{z} v_{F}}{\omega_{c}}\right) e^{i\left(l-l^{\prime}\right) \theta},
$$

leading to areal density and velocity perturbations

$$
\begin{gathered}
N_{\omega}^{L}=\int_{0}^{\infty} \int_{0}^{2 \pi} f_{\omega}^{L} \mathrm{~d} \theta p \mathrm{~d} p=\frac{\chi_{e} \varepsilon_{0}}{e} k_{z} \phi, \\
v_{z \omega, q}=\frac{1}{N_{0}^{0}} \int_{0}^{\infty} \int_{0}^{2 \pi} v_{z} f_{\omega}^{L} \mathrm{~d} \theta p \mathrm{~d} p=-\frac{e \phi \omega}{m * v_{F}^{2} k_{z}} S, \\
\chi_{e}=-\frac{N_{0}^{0} e^{2}}{k_{z} m * \varepsilon_{0} v_{F}^{2}} S, \quad S=4 \sum_{l} \frac{l^{2} \omega_{c}^{2} J_{l}^{2}\left(\frac{k_{z} v_{F}}{\omega_{c}}\right)}{\omega^{2}-l^{2} \omega_{c}^{2}},
\end{gathered}
$$

where $\omega_{c}=e B_{s} / m *, m *=\varepsilon_{F} / v_{F}^{2}, \chi_{e}$ is the electron susceptibility.

The jump condition on the normal component of displacement vector at $x=0$

$$
\left.\left.\frac{\partial \phi}{\partial x}\right]_{0^{+}}-\varepsilon_{g} \frac{\partial \phi}{\partial x}\right]_{0^{-}}=\frac{e}{\varepsilon_{0}} N_{\omega}^{L}
$$


on using $N_{\omega}^{L}$ from Eq. (8) gives the dispersion relation for magnetoplasmons

$$
1+\varepsilon_{g}+\chi_{e}=0, \quad \text { or } \quad 1+\varepsilon_{g}=\frac{4 N_{0}^{0} e^{2}}{m * \varepsilon_{0} k_{z} v_{F}^{2}} \sum_{l} \frac{l^{2} \omega_{c}^{2} J_{l}^{2}\left(\frac{k_{z} v_{F}}{\omega_{c}}\right)}{\omega^{2}-l^{2} \omega_{c}^{2}}
$$

This equation for one wave number offers many values of frequency, each corresponding to a magnetoplasmonic mode. The frequency of the $l$ 'th plasmonic mode lies between $l \omega_{c}$ and $(l+1) \omega_{c}$, where $l \geq 1$. There is no mode at $\omega<\omega_{c}$. For the first mode at long wavelengths, $k_{z} v_{F} / \omega_{c} \ll 1$ (small gyroradius limit) only $l=1,-1$ terms are important and Eq. (12) gives

$$
\omega^{2}=\omega_{c}^{2}+\frac{N_{0}^{0} e^{2} k_{z}}{m * \varepsilon_{0}\left(1+\varepsilon_{g}\right)} .
$$

This is equivalent of an upper hybrid wave in a plasma. ${ }^{1}$ For $\omega_{c}=0$, Eq. (14) reduces to the usual unmagnetized plasmon dispersion relation [cf. Eq. (18), Ref. 21], where frequency scales as $k_{z}^{1 / 2}$ and $\left(N_{0}^{0}\right)^{1 / 4}$.

We have solved the dispersion relation, Eq. (12), numerically for the following parameters: $\varepsilon_{g}=3, G \equiv N_{0}^{0} e^{2} / m * \varepsilon_{0} v_{F} \omega_{c}=22$ (corresponding to $N_{0}^{0}=10^{11} \mathrm{~cm}^{-2}, B_{s}=1$ Tesla or any multiple of this areal density when the magnetic field is reduced by the same factor, keeping $N_{0}^{0} / B_{s}$ constant). In Fig. 2, we have plotted the normalized frequency as a function of normalized wavenumber for four magnetoplasmonic modes. At $k_{z} \rightarrow 0$, the modes have frequencies near the harmonics of cyclotron frequency. As $k_{z} v_{F} / \omega_{c}$ rises, the frequencies rise. For the first mode, the frequency rises to a maximum of $\omega=1.7 \omega_{c}$ at $k_{z} v_{F} / \omega_{c} \approx 1$ and then declines, asymptotically to $\omega_{c}$ at large $k_{z} v_{F} / \omega_{c}$. For the second mode, $\omega$ rises from $2 \omega_{c}$ at $k_{z}=0$ to $2.7 \omega_{c}$ at $k_{z} v_{F} / \omega_{c} \approx 1.8$ and then falls off. For the third mode, $\omega$ rises from $3 \omega_{c}$, initially very gradually and then faster, attains a maximum $\omega=3.9 \omega_{c}$ at $k_{z} v_{F} / \omega_{c} \approx 2.5$ and then falls off. For the fourth plasmonic mode, maximum $\omega=4.9 \omega_{c}$ occurs at $k_{z} v_{F} / \omega_{c} \approx 3.1$. In Fig. 3, we have plotted the dispersion curves for $G=110$ (corresponding to $N_{0}^{0}=10^{12} \mathrm{~cm}^{-2}, B_{s}=2$ Tesla). At this higher $N_{0}^{0} / B_{s}$ ratio, the mode frequencies rise more rapidly with $k_{z} v_{F} / \omega_{c}$ and attain maximum values closer to the next harmonic. At $k_{z} v_{F} / \omega_{c} \gg l$, the $l$ 'th harmonic Bernstein mode has

$$
\omega \approx l \omega_{c}\left[1+\frac{2 N_{0}^{0} e^{2} J_{l}^{2}\left(\frac{k_{z} v_{F}}{\omega_{c}}\right)}{m * \varepsilon_{0} k_{z} v_{F}^{2}\left(1+\varepsilon_{g}\right)}\right] .
$$

\section{Mode Conversion in Graphene Ribbons}

We introduce a ribbon structure on graphene, effectively making the areal electron density to have a ripple. The total electron density is thus

$$
N_{0 T}^{0}=N_{0}^{0}+N_{q}, \quad N_{q}=N_{q 0} e^{i q z} .
$$

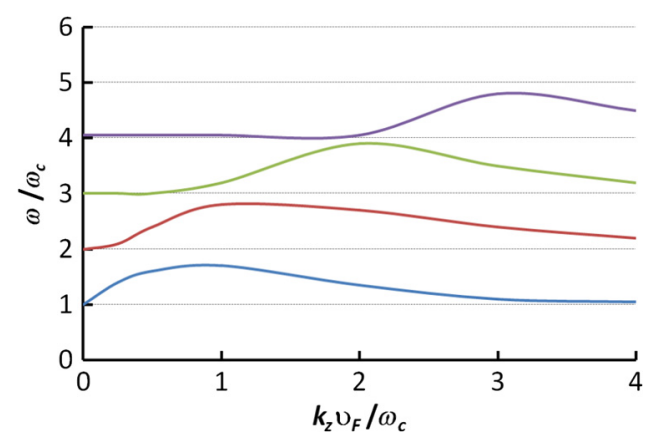

Fig. 2 Normalized frequency versus normalized wave number for magnetoplasmonic modes, originating at integer multiples of electron cyclotron frequency in graphene mounted on dielectric. The parameters are $G=N_{0}^{0} e^{2} / m * \varepsilon_{0} v_{F} \omega_{c}=22\left(N_{0}^{0}=10^{11} \mathrm{~cm}^{-2}, B_{s}=1\right.$ Tesla $), \varepsilon_{g}=3$. 


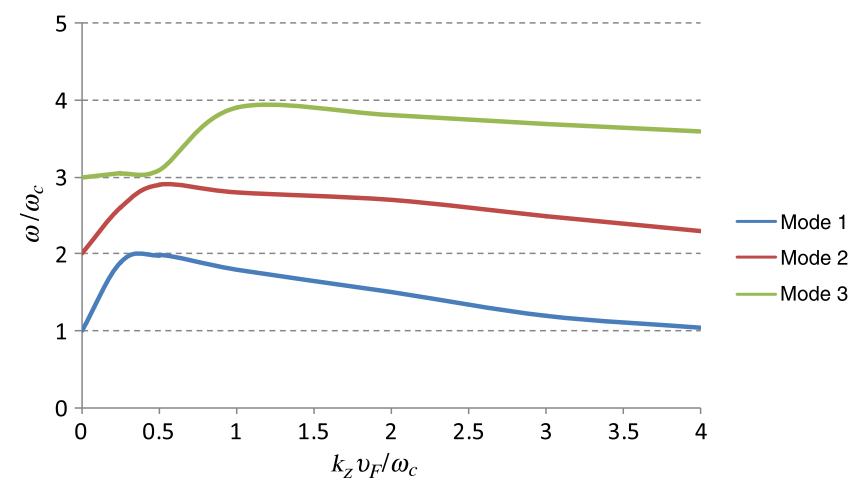

Fig. 3 Normalized frequency versus normalized wave number for magnetoplasmonic modes, originating at harmonics of cyclotron frequency in graphene for $G=110\left(N_{0}^{0}=10^{12} \mathrm{~cm}^{-2}\right.$, $B_{s}=2$ Tesla), $\varepsilon_{g}=3$.

Practically ribbon structures are step wise periodic, e.g., $N_{0 T}^{0}=N_{0}^{0}+\delta N$ for $n \lambda<z<n \lambda+a$ and $N_{0 T}^{0}=N_{0}^{0}$ for $n \lambda+a<z<(n+1) \lambda$, where $n$ is an integer, $a$ is the width of a ribbon, and $\lambda$ is the spatial periodicity. On carrying out Fourier series expansion of areal density in spatial harmonics of wave number $q=2 \pi / \lambda$, one obtains the amplitude of the first harmonic $N_{q 0}=(2 / \pi) N_{1} \sin (q a / 2)$. Higher harmonics have diminishingly smaller amplitudes.

A laser is normally incident on graphene from top

$$
\vec{E}_{0}=\hat{z} A_{0} e^{-i(\omega t+\omega x / c)}, \quad \vec{B}_{0}=\hat{y}\left(A_{0} / c\right) e^{-i(\omega t+\omega x / c)} .
$$

We choose $q$ such that it equals the magnetoplasmon wave number $k_{z}$ at frequency $\omega$ [cf., Eq. (12)]; $q \gg \omega / c$. The reflected and transmitted fields are

$$
\begin{aligned}
& \vec{E}_{0 R}=\hat{z} A_{0 R} e^{-i(\omega t-\omega x / c)}, \\
& \vec{B}_{0 R}=-\hat{y}\left(A_{0 R} / c\right) e^{-i(\omega t-\omega x / c)}, \\
& \vec{E}_{0 T}=\hat{z} A_{0 T} e^{-i\left(\omega t+\omega \eta_{g} x / c\right)}, \\
& \vec{B}_{0 T}=\hat{y}\left(A_{0 T} \eta_{g} / c\right) e^{-i\left(\omega t+\omega \eta_{g} x / c\right)},
\end{aligned}
$$

where $\eta_{g}=\varepsilon_{g}^{1 / 2}$ and we have presumed that the FR of polarization is small. The laser gives rise to perturbation in the electron distribution function $f_{0}^{L}$, which on solving the linearized Vlasov equation can be written as

$$
f_{0}^{L}=e A_{0 T} \frac{1}{p} \frac{\partial f_{0}^{0}}{\partial p} \int_{-\infty}^{t} p_{z}^{\prime} e^{-i \omega t^{\prime}} \mathrm{d} t^{\prime}=\frac{i e E_{0 z}}{2} \frac{\partial f_{0}^{0}}{\partial p}\left[\frac{e^{i \theta}}{\omega-\omega_{c}}+\frac{e^{-i \theta}}{\omega+\omega_{c}}\right],
$$

giving the drift velocity and surface current density

$$
v_{0 z}=\frac{1}{N_{0}^{0}} \int_{0}^{\infty} \int_{0}^{2 \pi} v_{z} f_{0}^{L} \mathrm{~d} \theta p \mathrm{~d} p=-\frac{i e \mathrm{E}_{0 z} \omega}{m *\left(\omega^{2}-\omega_{c}^{2}\right)}, \quad K_{0 z}^{L}=\frac{i N_{0}^{0} e^{2} E_{0 z} \omega}{m *\left(\omega^{2}-\omega_{c}^{2}\right)} .
$$

Since the laser wave vector has no component in the plane of electron gyration, the Larmor radius effects do not appear in electron response. The oscillatory velocity $v_{0 z}$ beats with the density ripple to produce an areal density perturbation, which on solving the equation of continuity, $\partial N_{\omega, q}^{N L} / \partial t+(1 / 2) \partial / \partial z\left(N_{q} v_{0 z}\right)=0$, turns out to be

$$
N_{\omega, q}^{N L}=\frac{q}{2 \omega} N_{q} v_{0 z} .
$$

Here, we have used the complex number identity $\operatorname{Re} A \cdot \operatorname{Re} B=(1 / 2) \operatorname{Re}[A \cdot B+A \cdot B *]$, where $\operatorname{Re}$ stands for the real part of the quantity and $*$ denotes the complex conjugate. This density perturbation acts as driver for the plasmonic wave. Let the potential of the plasmonic wave be $\phi$, 
given by Eq. (2). It creates linear density and velocity perturbations $N_{\omega}^{L}, \vec{v}_{\omega, q}$ given by Eqs. (8) and (9). obtains

Using $N_{\omega, q}^{L}, N_{\omega, q}^{N L}$ in the jump condition, Eq. (11) with $N_{\omega}^{L}$ replaced by $N_{\omega}^{L}+N_{\omega, q}^{N L}$, one

$$
D A=i \frac{N_{q 0} e^{2} A_{0 T}}{2 m * \varepsilon_{0}\left(\omega^{2}-\omega_{c}^{2}\right)}, \quad D=1+\varepsilon_{g}+\chi_{e} .
$$

The oscillatory velocity due to the magnetoplasmonic wave $\mathrm{v}_{z \omega, q}$ beats with the density ripple to produce a surface current density

$$
\vec{K}_{0}^{N L}=-\frac{1}{2} N_{q}^{*} e \vec{v}_{\omega, q}
$$

which acts as a source to influence the optical wave. Using this in the jump condition on the magnetic field

$$
\left.B_{0 y}\right|_{0^{+}}-\left.B_{0 y}\right|_{0^{-}}=\mu_{0}\left(K_{0 z}^{L}+K_{0 z}^{N L}\right),
$$

and employing the continuity of $E_{0 z}$ at $x=0$ one obtains

$$
\begin{gathered}
A_{0}+A_{0 R}=A_{0 T}, \\
A_{0}-A_{0 R}=\eta_{g} A_{0 T}+i \frac{N_{0}^{0} e^{2} \omega}{m * \varepsilon_{0} c\left(\omega^{2}-\omega_{c}^{2}\right)} A_{0 T}+\frac{N_{q 0}^{*} e^{2} \omega S}{2 m * \varepsilon_{0} c q v_{F}^{2}} A .
\end{gathered}
$$

Equations (23), (25), and (26) yield the amplitude of the transmitted laser field

$$
A_{0 T}=\frac{2 A_{0}}{1+\eta_{g}+i \frac{N_{0}^{0} e^{2} \omega}{m * \varepsilon_{0} c\left(\omega^{2}-\omega_{c}^{2}\right)}+i\left(\frac{N_{q 0}^{*} e^{2}}{m * \varepsilon_{0}}\right)^{2} \frac{\omega S}{4 c q v_{F}^{2} D\left(\omega^{2}-\omega_{c}^{2}\right)}} .
$$

The third term in the denominator corresponds to optical conductivity of graphene at the laser frequency. At frequencies one percent away from the cyclotron resonance, $\left(\omega-\omega_{c} \geq 0.01 \omega\right)$ this term is insignificant as compared to the first two terms. At cyclotron resonance, it is masked by collisions. However, around that point, the FR of polarization is significant and present formalism is not applicable.

The last term in the denominator of Eq. (26) corresponds to mode coupling of laser to magnetoplasmons. At exact phase matching [i.e., when $q$ equals the wave number of the plasmonic wave given by Eq. (12)], $D$ vanishes and the last term in the denominator of Eq. (26) overflows. However, in the present formalism, we have neglected damping of the plasmonic mode that may arise due to collisions. The damping of plasmons would arrest the resonance. As a phenomenological model of collisional effects, the right-hand side of the Vlasov equation may be replaced by $-\nu\left(f-f_{0}^{0}\right)$, where $\nu$ is the collision frequency. In the case of the upper hybrid mode, this leads to replacing, in the dispersion relation, $\omega^{2}$ by $\omega(\omega+i \nu)$ leading to damping rate $\omega_{i}=\nu / 2$. For the Bernstein modes, we may replace, in the expression for susceptibility or $S, \omega^{2}$ by $\omega(\omega+i \nu)$, giving an imaginary part to $D, D=D_{r}+i D_{i}$. At resonance, $D_{r}$ vanishes and $D_{i}$ can be written as

$$
D_{i} \approx \frac{4 N_{0}^{0} e^{2} \nu \omega}{m * \varepsilon_{0} k_{z} v_{F}^{2}} \sum_{l} \frac{l^{2} \omega_{c}^{2} J_{l}^{2}\left(\frac{k_{z} v_{F}}{\omega_{c}}\right)}{\left(\omega^{2}-l^{2} \omega_{c}^{2}\right)^{2}}
$$

From Eqs. (21) and (26), one may write the normalized plasmonic field amplitude and laser intensity transmission coefficient at the mode conversion point as 


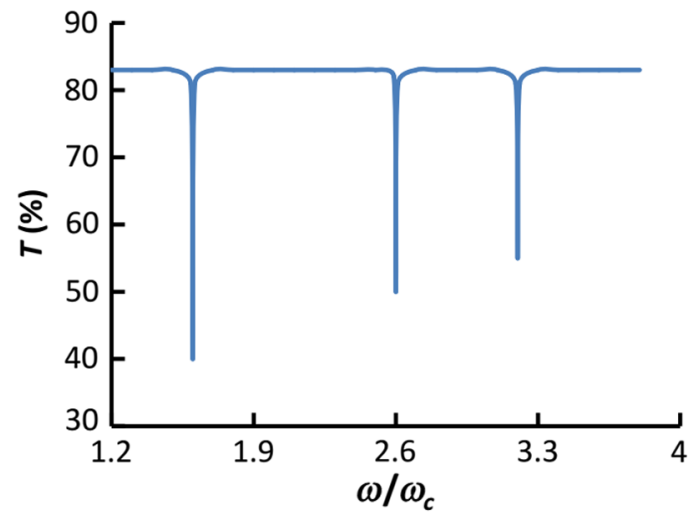

Fig. 4 Intensity transmission coefficient of laser normally impinged on graphene (with periodic ribbons of wave numbers $\left.q=\omega_{c} / v_{F}\right)$ as a function of normalized laser frequency. The parameters are: $G=22\left(N_{0}^{0}=10^{11} \mathrm{~cm}^{-2}, B_{s}=1\right.$ Tesla), $\varepsilon_{g}=3$. Dips at A, B, C refer to excitations of first, second, and third kinetic (Bernstein) magnetoplasmonic modes.

$$
\begin{gathered}
T=\left|\frac{A_{0 T}}{A_{0}}\right|^{2} \eta_{g}=\frac{4 \eta_{g}}{\left[1+\eta_{g}+\psi^{2}\right]^{2}+\left[\frac{N_{0}^{0} e^{2} \omega}{m^{*} \varepsilon_{0} c\left(\omega^{2}-\omega_{c}^{2}\right)}\right]^{2}}, \\
\left|\frac{q A}{A_{0}}\right|=\left|\frac{N_{q 0} e^{2} q}{m * \varepsilon_{0} D_{i}\left(\omega^{2}-\omega_{c}^{2}\right)}\right|\left(\frac{T}{\eta_{g}}\right)^{1 / 2}, \quad \psi=\frac{\left(N_{q 0} / N_{0}^{0}\right)^{2} G^{2} S \omega / q c}{4 D_{i}\left(\omega^{2} / \omega_{c}^{2}-1\right)},
\end{gathered}
$$

where $G \equiv N_{0}^{0} e^{2} / m * \varepsilon_{0} v_{F} \omega_{c}$. One may note that the amplitude of the plasmon electric field $|q A|$ may far exceed the laser field due to strong localization of plasmons. The fractional laser power going into the magnetoplasmonic mode is

$$
\eta=1-T-\left|\frac{A_{0 R}}{A_{0}}\right|^{2}=\frac{4 \psi}{\left(1+\eta_{g}+\psi\right)^{2}} .
$$

We have carried numerical calculations for the following parameters $N_{q 0} / N_{0 m}^{0}=0.5, \varepsilon_{g}=3$, $G=22, q v_{F} / \omega_{c}=1, \nu / \omega_{c}=2 \times 10^{-3}$ corresponding to $N_{0 m}^{0}=10^{11} \mathrm{~cm}^{-2}, m *=10^{-32} \mathrm{~kg}$, $q=1.6 \times 10^{5} \mathrm{~cm}^{-1}, \varepsilon_{F}=50 \mathrm{meV}, B_{s}=1$ Tesla, $\omega_{c}=1.6 \times 10^{13} \mathrm{rad} / \mathrm{s}$. We have plotted in Fig. 4 the intensity transmission coefficient of laser through graphene as a function of normalized laser frequency. At the plasmon resonance, corresponding to the excitation of the first magnetoplasmonic mode of frequency $\omega=1.7 \omega_{c}$, the transmission coefficient falls to $42 \%$. At the second plasmonic resonance $\omega=2.7 \omega_{c}$ corresponding to the excitation of the second plasmonic mode, the transmission coefficient is $52 \%$. At the third plasmon resonance, it is $60 \%$.

\section{Mode Conversion in Dielectric Grating}

Consider a dielectric grating of thickness $d$ sandwiched between graphene and the substrate. The relative permittivity of the grating region is

$$
\varepsilon_{r}=\varepsilon_{g 0}+\varepsilon_{q}, \quad \varepsilon_{q}=\varepsilon_{q 0} e^{1 q z}
$$

A laser is normally impinged on graphene with incident, reflected, and transmitted fields given by Eqs. (16) and (17). The driven plasmonic wave has potential given by Eq. (2). In the grating region, the Poisson equation can be written as

$$
-\varepsilon_{0} \varepsilon_{g 0} \nabla^{2} \phi=\rho^{N L}, \quad \rho^{N L}=-\frac{i}{2} q \varepsilon_{0} \varepsilon_{q} E_{0 z} .
$$

$\rho^{N L} d$ can be treated as effective surface charge density to excite the plasmonic mode. Equivalent areal electron density at $\omega, q$ is 


$$
N_{\omega, q}^{N L}=-\frac{d}{e} \rho^{N L}=\frac{i q d}{2 e} \varepsilon_{0} \varepsilon_{q} E_{0 z} .
$$

From the Maxwell's equation $\nabla \times \vec{B}=\mu_{0}(\vec{J}+\partial \vec{D} / \partial t)$, one may see that the last term, as a beat of the plasmonic wave field with the permittivity ripple, gives an effective surface current density at frequency $\omega$ and wave number $k_{z}=0$

$$
K_{0 z}^{N L}=-i \omega \varepsilon_{0} \frac{\varepsilon_{q}^{*}}{2}(-i q \phi) d
$$

With these expressions for $N_{\omega, q}^{N L}$ and $K_{0 z}^{N L}$, Eqs. (11) and (23) lead to

$$
D A=-i \frac{\mathrm{d} \varepsilon_{q}}{2} \varepsilon_{0} A_{0 T}, \quad A_{0}-A_{0 R}=\eta_{g} A_{0 T}+i \frac{N_{0}^{0} e^{2} \omega}{m * \varepsilon_{0} c\left(\omega^{2}-\omega_{c}^{2}\right)} A_{0 T}-\frac{\omega \varepsilon_{q}^{*} q d}{2 c} A,
$$

giving

$$
A_{0 T}=\frac{2 A_{0}}{1+\eta_{g}+i \frac{N_{0 m}^{0} e^{2} \omega}{m * \varepsilon_{0} c\left(\omega^{2}-\omega_{c}^{2}\right)}+i \frac{\left|\varepsilon_{q}\right|^{2} \omega q d^{2}}{4 c D}} .
$$

The transmission coefficient is given by Eq. (27) with $\psi$ given by

$$
\psi=\frac{\left|\varepsilon_{q}\right|^{2} \omega q d^{2}}{4 c \omega_{i} \partial D / \partial \omega} .
$$

The treatment is valid for $\varepsilon_{q 0} / \varepsilon_{g 0}<1, q d<1$. The effect of the dielectric grating is very similar to that of the density ripple.

\section{Discussion}

A transverse magnetic field opens up a variety of magnetoplasmonic modes in graphene. The lowest frequency mode, at wavelengths longer than the electron gyroradius, is sort of an upper hybrid mode; however, its frequency variation with wave number and electron density is very different from plasmas. Other modes have frequencies close to harmonics of cyclotron frequency. At shorter wavelengths, kinetic effects become important and mode frequencies rise with wave number, acquire maxima, and then fall off gradually. This behavior is similar to the one reported by Roldan et al. at high magnetic fields when quantum effects are important.

The magnetoplasmonic modes can be excited by laser via linear mode conversion, when the graphene layer is embedded with ribbons or a dielectric grating is employed between the substrate and graphene. For a given ripple wave number, the magnetic field helps tuning the process of laser mode conversion. Crassee et al. ${ }^{13}$ carried experiments with wrinkled surfaces and $p$-type graphene. They observed a dip in the laser transmission coefficient of magnitude comparable to what we get in Fig. 4. However, their variation of transmission coefficient with magnetic field does not reveal the discrete character as one would expect from mode conversion to Bernstein modes. The experiments need to minimize collisional damping of the modes.

The wide range of magnetoplasmonic modes offer new opportunities for active plasmonic devices at terahertz frequencies. One may envisage stimulated excitation of these modes by electron hole recombination under conditions of population inversion. Their frequencies can be tuned by magnetic field. The application of a surface ripple would convert the plasmonic modes into terahertz radiation emission.

The present treatment of mode conversion is limited to a low depth of amplitude modulation of ribbons, i.e., $N_{1} / N_{q 0}<1 / 2$ otherwise, a large number of spatial harmonics of plasmons are excited. ${ }^{22}$

\section{Acknowledgments}

The authors are thankful to Prof. Hao In for fruitful discussions. 


\section{References}

1. D. N. Basov et al., "Colloquium: graphene spectroscopy," Rev. Mod. Phys. 86, 959-994 (2014).

2. A. H. Castro Nato et al., "The electronic properties of graphene," Rev. Mod. Phys. 81, 109162 (2009).

3. T. Otsuji et al., "Graphene based devices in terahertz science and technology," J. Phys. D: Appl. Phys. 45, 303001 (2012).

4. N. M. R. Peres, "Colloquium: the transport properties of graphene: an introduction," Rev. Mod. Phys. 82, 2673-2700 (2010).

5. A. K. Geim, "Graphene: status and prospects," Science 324, 1530-1534 (2009).

6. A. K. Geim and K. S. Novoselov, "The rise of graphene," Nat. Mater. 6, 183-191 (2007).

7. S. D. Sarma et al., "Electronic transport in two-dimensional graphene," Rev. Mod. Phys. 83, 407-470 (2011).

8. E. McCann and M. Koshino, "The electronic properties of bilayer graphene," Rep. Prog. Phys. 76, 056503 (2013).

9. R. Roldan, J. N. Fuchs, and M. O. Goerbig, "Collective modes of doped graphene and a standard two-dimensional electron gas in a strong magnetic field: linear magnetoplasmons versus magnetoexcitons," Phys. Rev. B 80, 085408 (2009).

10. R. Roldan, M. O. Goerbig, and J. N. Fuchs, "Theory of Bernstein modes in graphene," Phys. Rev. B 83, 205406 (2011).

11. H. Yan et al., "Infrared spectroscopy of tunable Dirac terahertz magneto-plasmons in graphene," Nano Lett. 12, 3766-3771 (2012).

12. H. Yan et al., "Plasmonics of coupled graphene micro-structures," New J. Phys. 14, 125001 (2012).

13. I. Crassee et al., "Intrinsic terahertz plasmons and magnetoplasmons in large scale monolayer graphene," Nano Lett. 12, 2470-2474 (2012).

14. J. C. Martinez and M. B. A. Jalil, "Theory of giant Faraday rotation and Goos-Hänchen shift in graphene," Europhys. Lett. 96, 27008 (2011).

15. S. A. Mikhailov, "Plasma instability and amplification of electromagnetic waves in low dimensional electron systems," Phys. Rev. B 58(3), 1517-1532 (1998).

16. W. Lee and F. L. Degertekin, "Rigorous coupled-wave analysis of multilayered grating structures," J. Lightwave Technol. 22, 2359-2363 (2004).

17. W. Gao et al., "Excitation of plasmonic waves in graphene by guided-mode resonances," ACS Nano 6, 7806-7813 (2012).

18. V. Ryzhii, A. Satou, and T. Otsuji, "Plasma waves in two-dimensional electron-hole system in gated graphene heterostructures," J. Appl. Phys. 101, 024509 (2007).

19. K. G. Batrakov et al., "Plasmon polariton deceleration in graphene structures," J. Nanophotonics 6, 061719 (2012).

20. K. G. Batrakov and S. A. Maksimenko, "Graphene layered system as a terahertz source with tuned frequency," Phys. Rev. B 95, 205408 (2017).

21. E. H. Hwang and S. Das Sarma, "Dielectric function, screening, and plasmons in twodimensional graphene," Phys. Rev. B 75, 205418 (2007).

22. M. V. Krasheninnikov and A. V. Chaplik, "Radiative decay of two-dimensional plasmons," Sov. Phys. JETP 61, 75-77 (1985).

Chuan Sheng Liu is a plasma physicist credited with groundbreaking contributions to theory of laser plasma interaction and promoting international collaboration in higher education. He joined the University of Maryland in 1975 as a professor of physics and served twice as a department chair. He also served as a vice president for research and dean of the graduate school. From 2003 to 2006, he was a president of National Central University, Taiwan. Currently, he is now Master Emeritus of Chao Kuang Piu College.

Vipin K. Tripathi received his master's degree in physics from Agra University in 1967 and a PhD from IIT Delhi in 1971. In 1972, he joined the IIT faculty. In 1976, he moved to the University of Maryland and worked in the area of thermonuclear fusion, developing nonlinear theories of RF heating and current drive in tokamak. In 1983, he joined IIT Delhi as a professor of physics. He established a leading group in free electron laser and laser plasma interaction. 